\title{
Epidemiological, biochemicall, genetical aspects of pheochromocytomas and paragangliomas followed in CHU of Liège between 1993 and 2017
}

\author{
Petignot Sandrine ${ }^{1}$, Vroonen Laurent ${ }^{1}$, Hamoir Etienne ${ }^{2}$, Creemers Etienne ${ }^{3}$, Beckers Albert ${ }^{1}$ \\ 1 : Service d'Endocrinologie' $\mathrm{CHU}$ de Liège \\ 2 : Service de Chirurgie des glandes Endocrines, $\mathrm{CHU}$ de Liège \\ 3 : Service de Chirurgie Cardio-Vasculaire, $\mathrm{CHU}$ de Liège
}

\section{Objects}

Pheochromocytomas and paragangliomas (PPGLs) are rare tumors that are responsible for an inappropriate production of catecholamines. They are heritable in $40 \%$ of cases and can integrate different genetic syndroms. Thus, there are several interests to detect them precociously : avoid comorbidities due to the catecholamine hypersecretion, search syndroms and manage their related manifestations and screen the families. The objectives of this study were to separate PPGLs into 3 groups (pheochromocytomas, sympathic and parasympathic paragangliomas paragangliomas) and determinate for each group the proportion of germinal mutations. We were also interested in the type of catecholamine secretions and the correlation between the catecholamine secretion and the tumor size. Finally, we describe the relapsed and malignant forms and searched factors that had promoted them.

\section{Design of the study}

It's a retrospective study, based on 80 patients, operated in CHU of Liege between 1993 and 2017.

\section{Methods}

We had collected datas about age at diagnosis, genetic results, the presence of hypertension and the necessary number of anti-hypertensive medications before and after surgery. We classified the size of the tumors, their location and their features (bilateral tumors at diagnosis, relapsed and malignant tumors). We had also indicated the datas of 24hour urinary excretion of catecholamines.

\section{Results}

Regarding pheochromocytomas $(n=56)$, median age is 51 years (minimum : 11 ; maximum : 79). Three cases were bilateral at diagnosis $(5,37 \%)$, three cases relapsed $(5,37 \%)$ and two were malignant $(3,57 \%)$. We observed a correlation between secretion and tumor size for metanephrin $(p=0,0423)$ and normetanephrin $\left(p=6,18 \times 10^{-5}\right)$. Finally, we found 13 germinal mutations for 6 different genes among the 40 patients which had profited of a genetic screening $(32,50 \%)$.

Regarding sympathic paragangliomas $(n=8)$, median age is 43,5 years (minimum : 28 ; maximum : 71). There were two relapsed cases $(25 \%)$ and four malignant cases $(50 \%)$. We did not observed correlation between secretion and tumor size, neither for metanephrin $(p=$ $0,121)$ nor normetanephrin $(p=0,579)$. Among 6 patients which profited of a genetic screening, we found 2 germinal mutations into 2 different genes $(33,33 \%)$. 
Regarding parasympathic paragangliomas $(n=17)$, median age is 54 years (minimum:20 ; maximum : 88). Two cases had relapsed $(11,76 \%)$ and one case was malignant $(5,88 \%)$. Their location concerned tympanic glomus $(52,94 \%)$, carotid body $(35,29 \%)$, jugular glomus $(5,88 \%)$ and vagal nerve $(5,88 \%)$. A genetic screening had been realised in one patient and was negative.

\section{Conclusions}

With our study, we have shown the correlation between tumor size and catecholaminergic secretion in our pheochromocytoma group. We have also illustrated the link between genetics and development of bilateral/relapsed PPGLs, expecially in young patients. A genetic screening had been done among 47 patients and shown germinal mutations in $29,78 \%$ of cases. Finally, one of these patients had a syndrome described for the first time few months ago, characterized by a pheochromocytoma and a pituitary adenoma due to a deletion in MAX gene. 